\title{
Simulation of Scattering of Bending Characteristics of FRCC Based on Bridging Law Considering Fiber Distribution
}

\author{
Toshiyuki Kanakubo ${ }^{1}$, Keisuke Watanabe ${ }^{2}$ and Yuriko $\mathrm{Ozu}^{3}$
}

${ }^{1}$ Department of Engineering Mechanics and Energy, University of Tsukuba, 1-1-1, Tennohdai, Tsukuba, 305-8573, Japan; PH +81(29)853-5045; FAX +81(29)853-5207; email: kanakubo@kz.tsukuba.ac.jp

${ }^{2}$ Central Japan Railway Company, JR Central Towers, 1-1-4, Meieki, Nakamura-ku, Nagoya, 450-6101, Japan

${ }^{3}$ Department of Engineering Mechanics and Energy, University of Tsukuba, 1-1-1, Tennohdai, Tsukuba, 305-8573, Japan; PH +81(29)853-5462; FAX +81(29)853-5207; email: s1620871@u.tsukuba.ac.jp

\begin{abstract}
It is well known that tensile and bending characteristics of fiber-reinforced cementitious composite (FRCC) are influenced by fiber orientation and distribution. In this study, a visualization simulation is conducted using sodium silicate solution (known as water glass) to observe the flow patterns of the fibers in the beam specimen. The results of the visualization simulation are discussed mainly for the distribution of the position of each single fiber. In this study, based on the visualization results, Poisson distribution for expressing the position of fibers is adopted to calculate the bridging law (tensile stress - crack width relationship), in which the pullout properties of the single fiber are considered. The influence of fiber orientation is also considered in the calculation using the elliptic function characterized by the principal orientation angle and the orientation intensity. The scattering of maximum tensile stress (bridging strength) can be confirmed by Monte Carlo simulation (MCS), in which the fiber distribution following Poisson distribution is considered. The calculated bridging law is modeled by trilinear model, and section analysis is conducted to compare with the bending test results using polyvinyl alcohol (PVA) fiber. The possibility to evaluate the variation of bending strength can be found out by considering fiber distribution.
\end{abstract}

Keywords: Fiber distribution, Visualization, Poisson distribution, Monte Carlo simulation, Bridging law

\section{INTRODUCTION}

It is well known that tensile and bending characteristics of fiber-reinforced cementitious composite (FRCC) are influenced by fiber orientation and distribution. Concerning fiber orientation, many researchers have studied the effects of fiber orientation on the mechanical characteristics of FRCC, including fiber-reinforced concrete (FRC). The scheme of the current approach to evaluate the fiber orientation has considered the casting method, fresh-state properties, flow, vibration, and 
formwork geometry (e.g., Laranjeira, et al. 2012). Even in case of strain-hardening cement composite (SHCC), casting direction influences the fiber orientation, and vertical casting in tension test specimens causes degradation of tensile strength and deformation capacity of SHCC (Kanakubo 2006). Concerning fiber distribution/ dispersion, on the other hand, there are a few studies (Kasperkiewicz 1977, Stroeven 1979) that researched the quantitative evaluation of fiber distribution in steel fiberreinforced concrete (SFRC). In these studies, X-ray system was used to take roentgenographs of steel fibers in SFRC. However, it is difficult to use X-ray system to investigate the distribution of polymeric fibers such as polyethylene (PE) and polyvinyl alcohol (PVA) fibers those are commonly used for SHCC. The authors visualized PVA fibers in SHCC using CT scan system (Asano and Kanakubo 2013). The fiber orientation angle was obtained from the visualized images and the fiber orientation angle distribution was evaluated by probability density function using elliptic function. However, the region of visualized images taken by CT scan system is limited and the evaluation of fiber dispersion is difficult from those images.

In this study, a visualization simulation is conducted using sodium silicate solution (water glass) to observe the flow patterns of the fibers in the beam specimen. The results of the visualization simulation are discussed mainly for the distribution of the position of each single fiber. Based on the visualization results, Poisson distribution for expressing the position of fibers is adopted to calculate the bridging law (tensile stress - crack width relationship). The scattering of maximum tensile stress (bridging strength) is discussed based on the results of Monte Carlo simulation (MCS), in which the fiber distribution following Poisson distribution is considered. The calculated bridging law is modeled by trilinear model, and section analysis is conducted to compare with the bending test results using PVA fiber.

\section{VISUALIZATION SIMULATION USING WATER GLASS}

In order to observe the flow patterns of fibers in matrix, a visualization simulation using water glass is performed. The simulation method is generally same with one in the previous study (Kanakubo, et al. 2016).

Outline of visualization simulation. Water glass has high viscosity, and it is colorless and transparent. In regards to the practical use of SHCC, the rheology of mortar matrix before mixing the fiber was inspected using the flow time using the funnel (Kanda, et al. 2006). The flow time of water glass was controlled by adding pure water in an effort to attain the same flow time of mortar matrix as that of the target SHCC. Black-colored "target fibers" made from nylon were added to the matrix to simplify the image analysis. The density, diameter and length of the target fiber is $1.14 \mathrm{~g} / \mathrm{cm}^{3}, 0.24 \mathrm{~mm}$ and $12 \mathrm{~mm}$, respectively. The length of target fiber was set to same length of used PVA fiber in the previous study (Ozu, et al. 2016). The volume fraction of the target fibers was set to $0.05 \%$ based on empirical trial-anderror results. The influence of the differences of the volume fraction in visualization simulation can be referred in the previous study (Kanakubo, et al. 2016).

The simulation situations and an example of photos are shown in Figure 1. The mold was constructed with transparent acrylic plates. The cross section of the mold was 
$180 \times 280 \mathrm{~mm}$ and the total length was $980 \mathrm{~mm}$. Water glass solution was poured into the mold using a bucket from the one end of the mold. After pouring, photos of the matrix surfaces were taken using digital cameras at in-plane resolution of $6000 \mathrm{x}$ 4000 pixels. The pouring of the matrix and taking photos were carried out by 4 times.
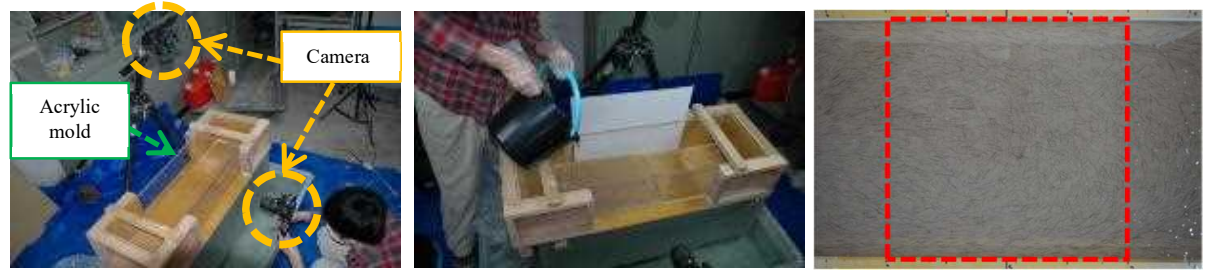

Figure 1. Visualization simulation using water glass.

Evaluation of fiber distribution. The obtained photos were cropped to include only the target region of $280 \times 280 \mathrm{~mm}$ square as shown in the right photo in Figure 1. The procedure of the image analysis is shown in Figure 2. After the cropping, the image was binarized and the noise was filtered (Figure 2 (b)). RGB values of the pixel data were read with position coordinates $\left(x_{i}, y_{i}\right)$, and the sequences of black-colored pixels were grouped and labeled. The averaged position coordinates $\left(x_{g}, y_{g}\right)$ of each group of black-colored pixels were calculated, and these coordinates were set to the coordinates of the centroid of each fiber (red points in Figure 2 (c)). The numbers of red points in separated region in the $x$-direction (longitudinal axis of the mold) with 1 $\mathrm{mm}$ interval were counted (Figure 2 (d)). After that, histograms for the numbers of fibers were drawn.

The histograms are shown in Figure 3. The average and standard deviation of the numbers of fibers are listed in Table 1 . The averages of the numbers of fibers range from 6.0 to 7.7. The standard deviations are $2.6-2.8$ for all pouring. Poisson distribution gives a discrete probability distribution that expresses the probability of events occurring in a fixed interval of time or space if these events occur independently. Figure 3 also describes Poisson distribution with the average

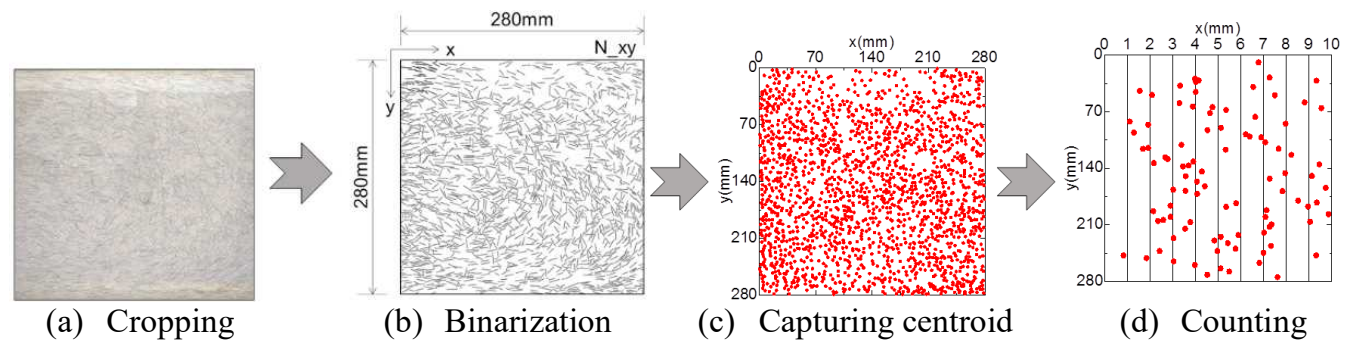

Figure 2. Procedure of image analysis.

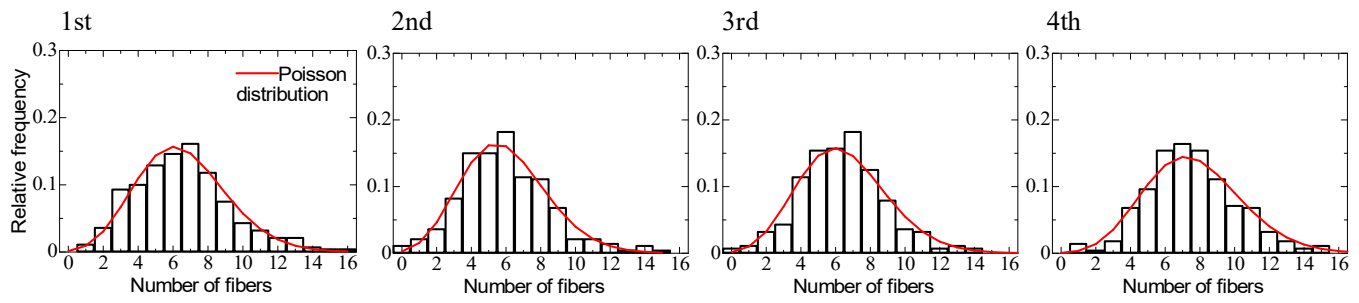

Figure 3. Histogram of number of fibers. 
Table 1. Average and STDV of number of fibers.

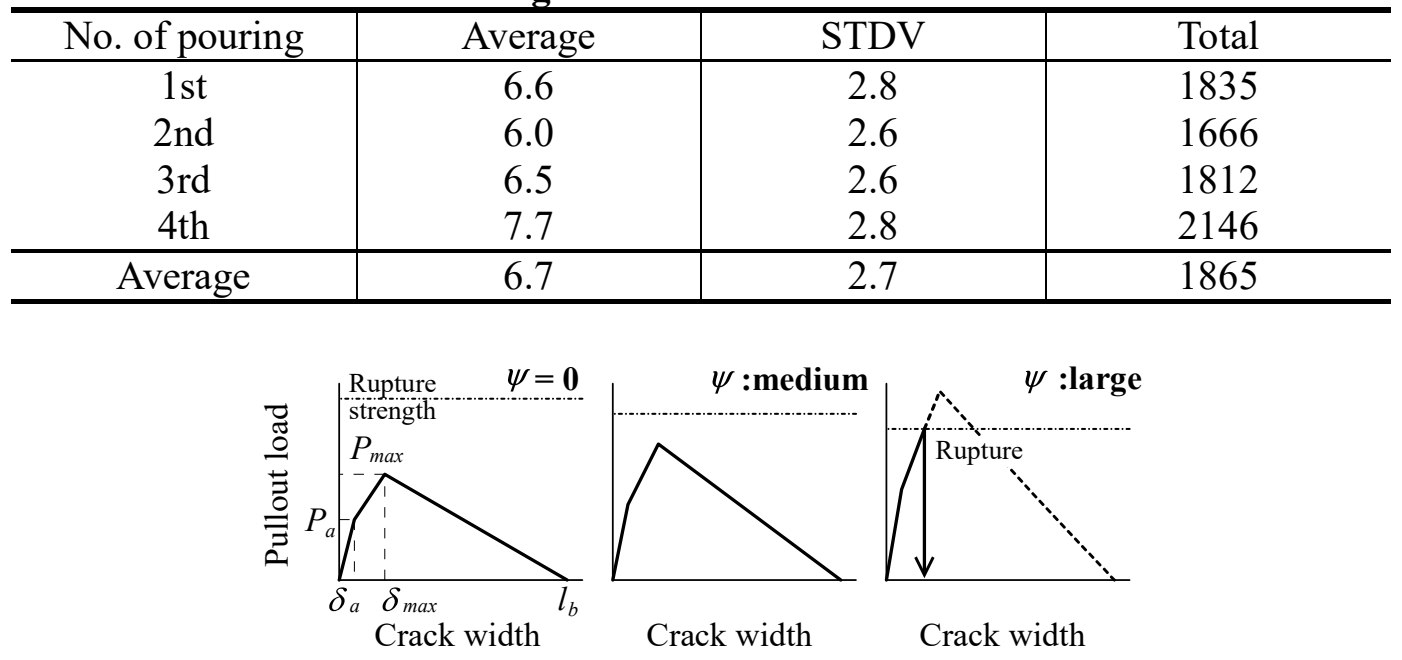

Figure 4. Trilinear model for pullout load of single PVA fiber.

occurrence number of 6.7 by solid line. As seen in Figure 3, Poisson distribution well expresses the number of occurrence which corresponds to the number of fibers in the target region.

\section{CALCULATION OF BRIDGING LAW CONSIDERING FIBER DISTRIBUTION}

Calculation method of bridging law. Calculation method of bridging law (tensile stress - crack width relationship) is generally same with one in the previous study (Kanakubo, et al. 2016). Calculation formula that gives tensile force (bridging force) is given by Eq.(1) as the summation of pullout force of each fiber.

$$
\begin{aligned}
& P_{\text {bridge }}=\sum_{h} \sum_{j} \sum_{i} P_{i j}(\delta, \psi) \cdot p_{x y}\left(\theta_{i}\right) \cdot p_{z x}\left(\phi_{j}\right) \cdot p_{x}\left(y_{h}, z_{h}\right) \cdot \Delta \theta \cdot \Delta \phi \cdot(\Delta y \cdot \Delta z) \\
& \left.P=P_{p u l l} \cdot e^{f \cdot \psi}<P_{r u p} \cdot e^{-f^{\prime} \cdot \psi} \text { (once exceeded, } P=0\right)
\end{aligned}
$$

Where, $P_{\text {bridge }}$ is bridging force (= total of pullout load), $P$ is pullout load of a single fiber, $P_{\text {pull }}$ is pullout load of a single fiber at a zero fiber angle, $P_{\text {rup }}$ is pullout load of a single fiber at rupture at a zero fiber angle, $f$ is snubbing coefficient, $f$ ' is fiber strength reduction factor, $p_{x y}, p_{z x}$ are probability based on elliptic distribution, $p_{x}$ is probability of fiber distribution along $x$-axis, $\psi$ is fiber angle to $x$-axis, $\theta$ is angle between $x$-axis and projected line of the fiber to $x-y$ plane, and $\phi$ is angle between $x$ axis and projected line of the fiber to $z-x$ plane.

Trilinear model for pullout load - crack width (determined from slip-out displacement) relationship of single PVA fiber, in which the snubbing effect and rupture strength degradation are considered, is adopted as shown in Figure 4. In Eq.(1), $p_{x y}$ and $p_{z x}$ express the probability density function (PDF) for fiber orientation, based on elliptic distribution characterized by the principal orientation angle, $\theta_{r}$, and the orientation intensity, $k$ (Kanakubo, et al. 2016). When the fibers orient perfectly 
randomly, $k$ becomes equal to 1 . When the fibers show an increased directional orientation toward $\theta_{r}$, the value of $k$ is larger than 1 . In contrast, when the fibers orient perpendicularly with respect to $\theta_{r}$, the value of $k$ is smaller than 1 . Figure 5 shows examples of elliptic distribution, and Table 2 presents the values for trilinear model, that is adopted in the previous study (Ozu, et al. 2016), in which the bending characteristics of PVA FRCC were investigated.

In this study, the centroid position of each fiber in $x$-direction is given following the probability of $p_{x}$, that is estimated by Poisson distribution. The average occurrence number in Poisson distribution is set to 6.7 based on the result of visualization simulation. Figure 6 shows the procedure of occurrence distributing of fiber centroid, following Poisson distribution. From Poisson distribution, the occurrence number and corresponding probability is estimated. The number of elements, that include the estimated occurrence number, is calculated as the total number of elements multiplied by estimated probability. In Figure 6 , the total number of elements is set to 20 . The

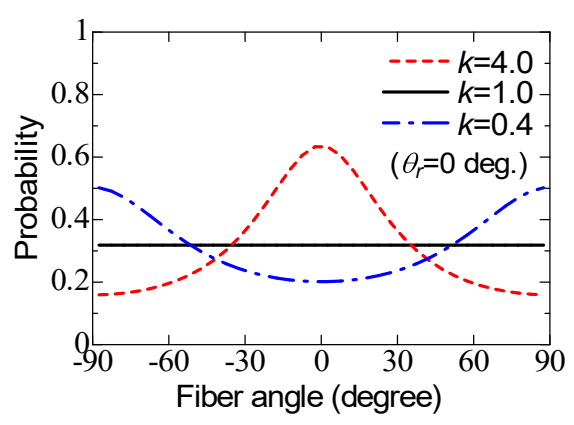

Figure 5. Elliptic distribution
Table 2. Used values for trilinear model.

\begin{tabular}{c|c}
\hline Item & Value \\
\hline First peak load, $P_{a}(\mathrm{~N})$ & 1.5 \\
Crack width at $P_{a}, \delta_{a}(\mathrm{~mm})$ & 0.2 \\
Maximum load, $P_{\max }(\mathrm{N})$ & 3.0 \\
Crack width at $P_{\max }, \delta_{\max }(\mathrm{mm})$ & 0.45 \\
Fiber strength, $\sigma_{f u},(\mathrm{MPa})$ & 569 \\
Snubbing coefficient, $f$ & 0.5 \\
Fiber strength reduction factor, $f$ & 0.3 \\
Fiber length, $l_{f}(\mathrm{~mm})$ & 12 \\
\hline
\end{tabular}

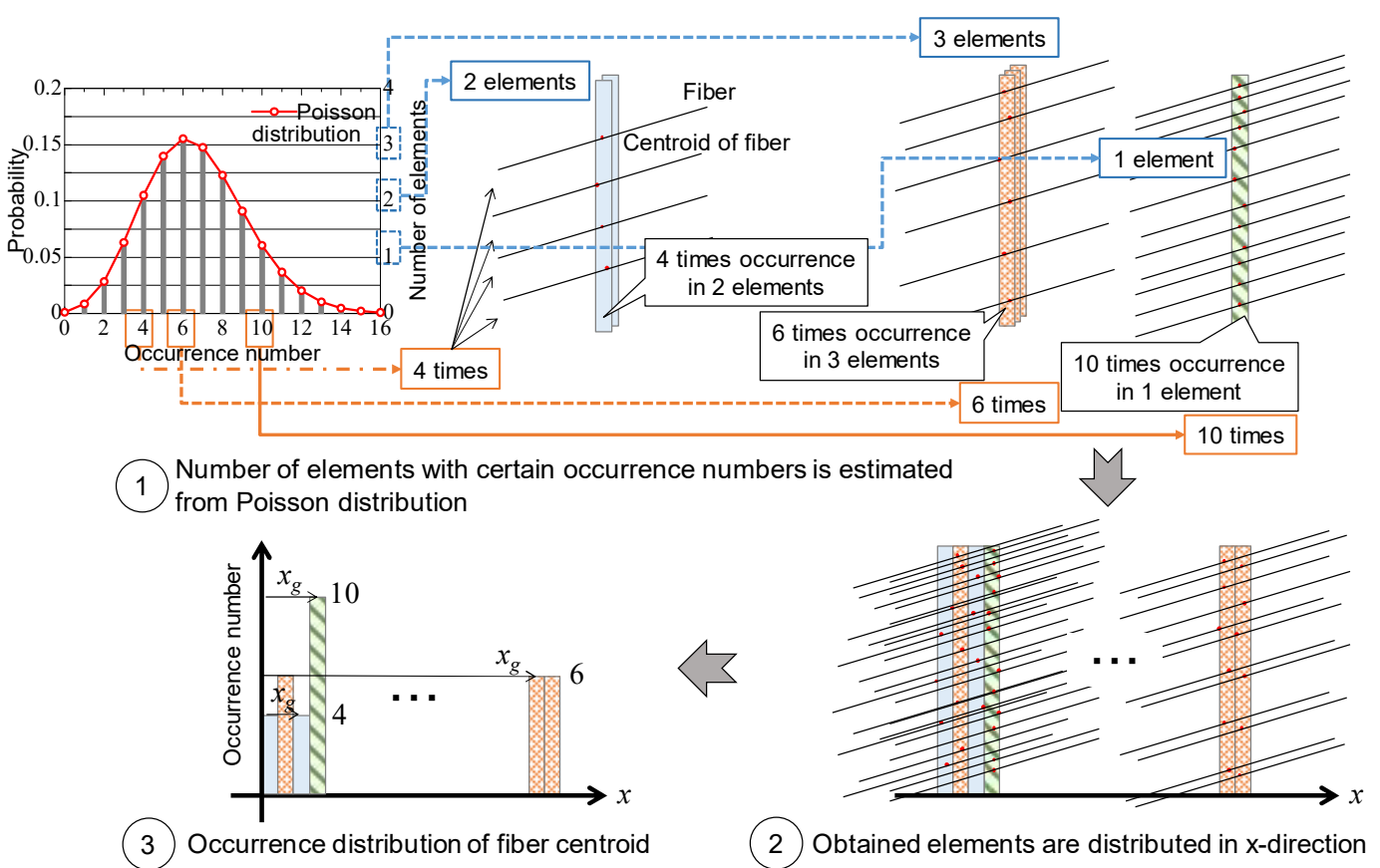

Figure 6. Procedure of occurrence distributing of fiber centroid. 
obtained elements are arbitrarily distributed in $x$-direction. At last, the occurrence distribution of the elements in $x$-direction is obtained, and the elements include the occurrence numbers for centroid of fibers. By this procedure, fiber distribution is assumed based on Poisson distribution. Fiber distribution can be changed by distributing method of the elements in $x$-direction.

By above mentioned calculating method, calculated bridging law varies by distributing method of the elements. It is considered that the maximum bridging performance can be obtained when the number of fibers which have larger embedded length (centroid of fiber position locates near crack surface, i.e., $x_{g}$ is near to 0 ) is large. In contrast, bridging performance becomes lower when the number of fibers which have shorter embedded length (centroid of fiber position locates far from crack surface, i.e., $x_{g}$ is large) is large. Figure 7 shows these typical cases of occurrence distribution (maximum or minimum distribution), and corresponding calculated bridging law. In this calculation, the total number of elements is set to 100 .
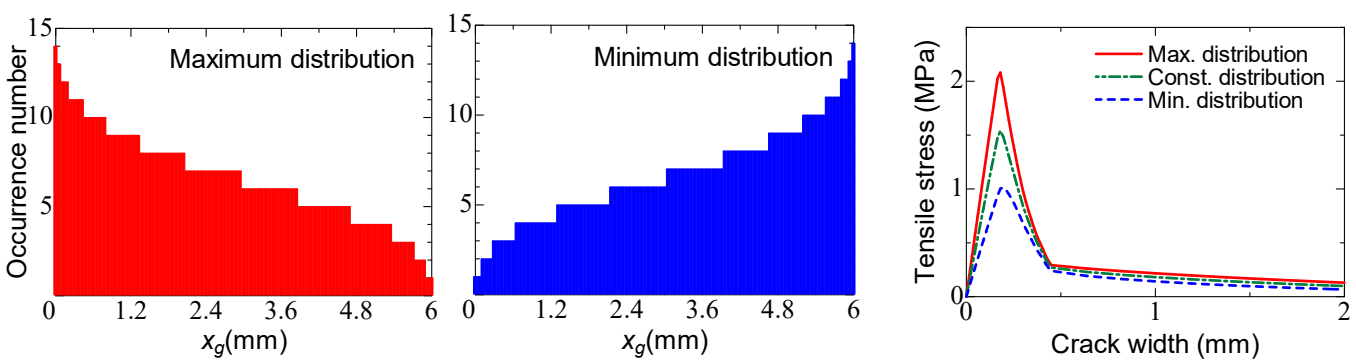

Figure 7. Maximum and minimum bridging law and their distributions.

Monte Carlo simulation to investigate the scattering of bridging law. The calculated bridging law varies by distributing method of the elements. The Monte Carlo simulation (MCS) is conducted to investigate the scattering of bridging law. The method of elements distributing is arbitrary, so the elements are distributed randomly. Figure 8 shows two examples of the distribution and corresponding calculated bridging law. This simulation is performed 3000 times for each orientation intensity, $k(0.4,1.0$, and 4.0$)$, to evaluate the scattering of bridging law.

Figure 9 shows the simulation results. The bridging strength is defined as the maximum tensile stress in bridging law. All the simulated bridging strengths exist among the calculation results by the maximum distribution and the minimum distribution shown in Figure 7. The simulation results of the bridging strength are summarized in Table 3. Standard deviation of the bridging strength ranges from 0.11
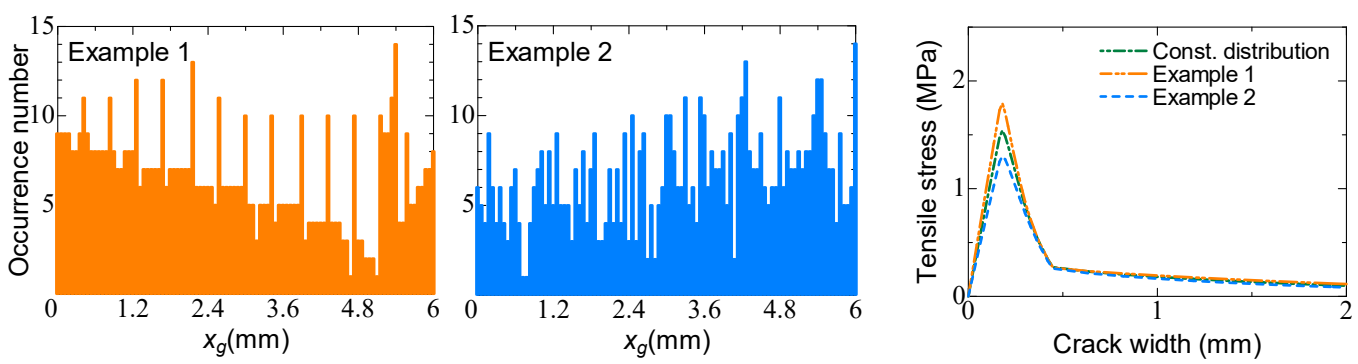

Figure 8. Example of occurrence distribution and calculated bridging law. 

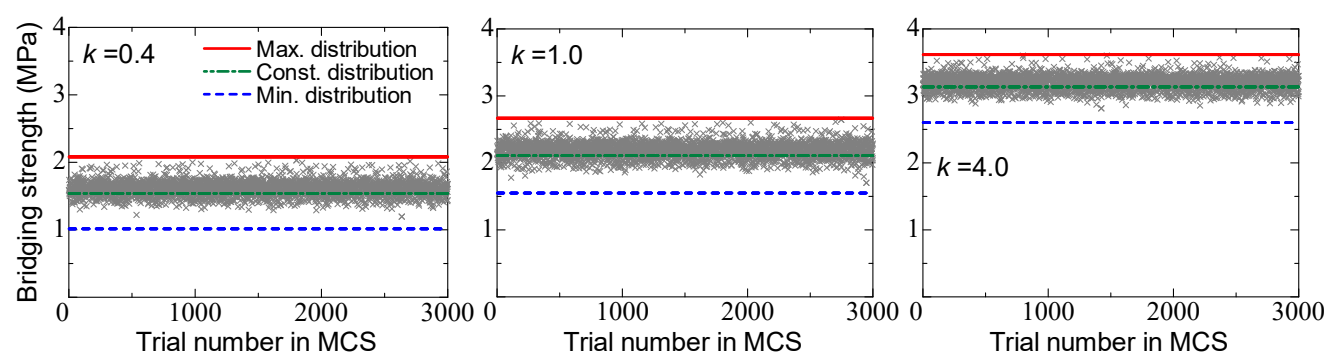

Figure 9. Bridging strength obtained by MCS.

Table 3. Bridging strength obtained by Monte Carlo simulation.

\begin{tabular}{|c|c|c|c|c|c|c|c|}
\hline \multirow{2}{*}{$\begin{array}{c}\text { Orient. } \\
\text { intensity }\end{array}$} & \multicolumn{4}{|c|}{ Monte Carlo simulation (MPa) } & \multirow{2}{*}{$\begin{array}{c}\text { Const. } \\
\text { dist. }\end{array}$} & \multirow{2}{*}{$\begin{array}{l}\text { Max. } \\
\text { dist. }\end{array}$} & \multirow{2}{*}{$\begin{array}{l}\text { Min. } \\
\text { dist. }\end{array}$} \\
\hline & Ave. & STDV & Max. & Min. & & & \\
\hline 0.4 & 1.61 & 0.11 & 2.03 & 1.19 & 1.54 & 2.08 & 1.01 \\
\hline 1.0 & 2.19 & 0.12 & 2.64 & 1.70 & 2.11 & 2.67 & 1.55 \\
\hline 4.0 & 3.20 & 0.11 & 3.60 & 2.81 & 3.13 & 3.62 & 2.60 \\
\hline
\end{tabular}

to $0.12 \mathrm{MPa}$. While the average of bridging strength is strongly affected by orientation intensity, it does not have large influence to scattering of the strength.

\section{EVALUATION OF VARIATION OF BENDING STRENGTH OF PVA FRCC}

Section analysis. The authors have tested bending characteristics of PVA FRCC in previous study (Ozu, et al. 2016). In that study, total of 18 specimens with $40 \times 40 \mathrm{~mm}$ cross-sectional dimension and 13 specimens with $100 \times 100 \mathrm{~mm}$ dimension were subjected to 4-point bending test. All specimens were manufactured by same mix proportion of PVA FRCC. The maximum bending stresses (defined as maximum bending moment divided by section modulus) observed in bending test are compared with those calculated by section analysis.

The left figure in Figure 10 shows the constitutive model of PVA FRCC for section analysis. The parabolic model is adopted in compression side, and the trilinear model is chosen for tension side to express the bridging law. The number of modeled bridging law is five, i.e., the bridging strength calculated by the maximum distribution (Max), and minimum distribution (Min), averaged strength obtained by MCS (MCSave), and MCSave $\pm 3 \sigma(\sigma$ : STDV) to express $99 \%$ confidence limits. The strain in tension side is converted from crack width to bridging law using average crack spacing observed in bending test.
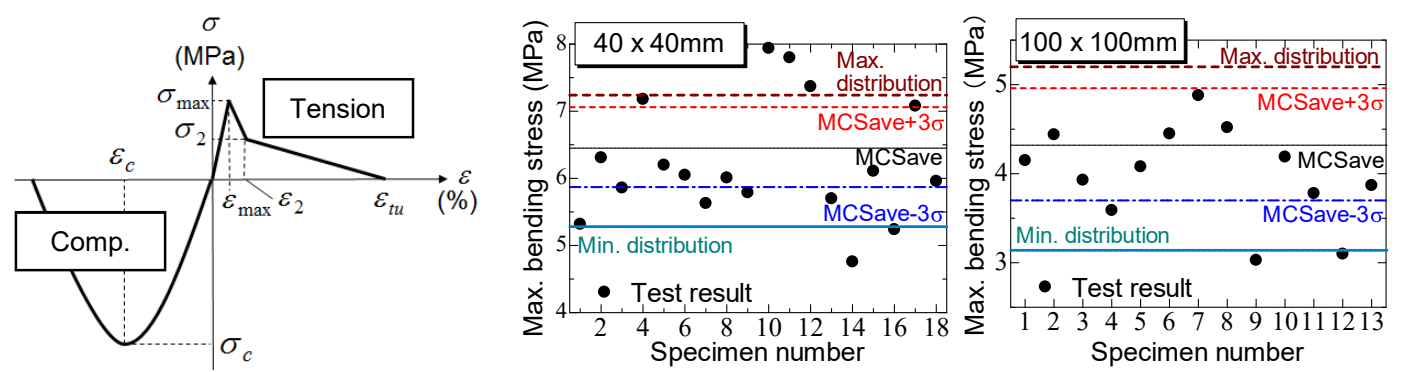

Figure 10. Model for section analysis and analysis results. 
Comparison of section analysis with test results. Figure 10 shows maximum bending stresses observed in bending test and calculated ones by section analysis. The orientation intensity for the specimens with $40 \times 40 \mathrm{~mm}$ section is set to 4.0 , and that for the specimens with $100 \times 100 \mathrm{~mm}$ section is 1.0 as similar to the previous study. In case of $40 \times 40 \mathrm{~mm}$ specimens, 6 test results exist in the region of confidence limits. Similarly, 10 test results of $100 \times 100 \mathrm{~mm}$ specimens exist in that region. It is considered that there is a possibility to evaluate the variation of bending strength by considering fiber distribution.

\section{CONCLUSION}

Based on the visualization results, Poisson distribution for expressing the position of fibers is adopted to calculate the bridging law. The scattering of maximum tensile stress (bridging strength) is evaluated by Monte Carlo simulation. Standard deviation of the bridging strength ranges from 0.11 to $0.12 \mathrm{MPa}$, those correspond to $4 \%-7 \%$ average strength. The calculated bridging law is modeled by trilinear model, and section analysis is conducted to compare with the bending test results. The possibility to evaluate the variation of bending strength can be found out by considering fiber distribution.

\section{REFERENCES}

Asano, K. and Kanakubo, T. (2013). "Study on the Effect of Fiber Orientation to Tensile Behavior of HPFRCC”, AIJ J. Struct. Constr. Eng., Vol.78, No.692, pp.1673-1678.

Kanakubo, T. (2006). "Tensile Characteristics Evaluation Method for Ductile FiberReinforced Cementitious Composites", JCI Journal of Advanced Concrete Technology, Vol.4, No.1, pp.3-17.

Kanakubo, T., Miyaguchi, M., and Asano, K. (2016). "Influence of Fiber Orientation on Bridging Performance of Polyvinyl Alcohol Fiber-Reinforced Cementitious Composite", ACI Materials Journal, Vol.113, No.2, pp.131-141.

Kanda, T., et al. (2006). "Full Scale Processing Investigation for ECC Pre-Cast Structural Element", Journal of Asian Architecture and Building Engineering, Vol.5, No.2, pp.333-340.

Kasperkiewicz, J. (1977). "Fibre spacing in steel fibre reinforced composites", Materials and Structures, Vol. 10, No. 1, pp.25-31.

Laranjeira, F., Aguado, A., Molins, C., Grünewald S., Walraven, J., and Cavalaro, S. (2012). "Framework to predict the orientation of fibers in FRC: A novel philosophy", Cement and Concrete Research, 42, pp.752-768.

Ozu, Y., Watanabe, K., Yasojima, A., and Kanakubo, T. (2016). "Evaluation of Size Effect in Bending Characteristics of DFRCC Based on Bridging Law", 7th International Conference of Asian Concrete Federation, 3. Concrete structures, Paper No.32.

Stroeven, P. (1979). "Morphometry of fibre reinforced cementitious materials Part I: Efficiency and spacing in idealized structures", Materiaux et Construction, vol. 12, No.1, pp.9-20. 